\title{
Ultrasonic pitch and catch technique for non-destructive testing of reinforced concrete slabs
}

\author{
Wael A. Zatar ${ }^{1,2}$, Hai D. Nguyen ${ }^{1,2^{*}}$ (D) and Hien M. Nghiem²
}

\begin{abstract}
This study aims at evaluating reinforced concrete (RC) bridge elements using ultrasonic pitch and catch (UPC) nondestructive testing (NDT) technique. A validation reinforced concrete slab with two embedded layers of rebars and artificial defects (voids, honeycombs, and debondings) was designed and tested. A commercial UPC NDT device (hereafter called "UPC device"), which is based on the ultrasonic shear-wave test method using dry-point-contact transmitting and receiving transducers in a "pitch-catch" configuration, was used to map internal defects of the validation RC slab. The recorded data from the UPC device was analyzed using a modified synthetic aperture focusing technique (SAFT). A software was developed to reconstruct 2-D images of the RC slab cross-sections using novel signal filtering and processing techniques. The results revealed that the 2-D image reconstructed from the developed software accurately exhibited locations and horizontal dimensions of the steel rebars, voids, and debondings. In addition, the developed software was capable to provide much higher resolution and sharper images of the anomalies inside the RC slab compared to the UPC device's proprietary imaging software.
\end{abstract}

Keywords: Ultrasonic pitch and catch (UPC), Reinforced-concrete slabs, Artificial defects, Voids, Honeycombs, Debondings, Modified SAFT (synthetic aperture focusing technique), Signal filtering and processing

\section{Introduction}

According to the 2017 infrastructure report card, America's infrastructure (e.g., bridges, rail, roads, dams and levees, transit, energy, and aviation) was rated $\mathrm{D}+$, which is interpreted as poor to fair conditions. This rating poses a serious concern as a large portion of the nation's infrastructure exhibited significant deterioration with a strong risk of failure. For bridge structures, approximately $40 \%$ of the total 614,387 bridges in the United States is equal to or over 50 years old. In 2016, about $9.1 \%$ of the nation's bridges (56,007 bridges) were structurally deficient (i.e., bridges that require significant maintenance, rehabilitation, or replacement) and 13.6\%

\footnotetext{
* Correspondence: nguyenhai@marshall.edu

'Advanced Material and Testing Laboratory, College of Engineering and Computer Sciences (CECS), Marshall University, One John Marshall Drive, Huntington, West Virginia 25755, USA

${ }^{2}$ Marshall University Research Corporation, Marshall University, One John Marshall Drive, Huntington, West Virginia 25755, USA
}

of the U.S. bridges were considered functionally obsolete (i.e., bridges that have the low load-carrying capacity and do not meet current engineering standards/specifications). The federal government estimates that $\$ 123$ billion are required to address the nation's backlog of bridge rehabilitation projects [4]. Proper inspection, maintenance, rehabilitation, or replacement of these bridges, therefore, play critical roles in the nation's economy and to the safety of the traveling public.

Visual inspection is known to be a fast, convenient, inexpensive, versatile, and simple testing technique for in-situ inspection of concrete structures. It, however, typically limits the inspector to examine anomalies/defects/discontinuities on the surface only and rely heavily on subjective assessments, which may significantly differ from one inspector expert to another [13, 26]. Accordingly, more reliable, rapid, and cost-effective testing methods are necessary to optimize the rehabilitation and

\section{Springer Open}

๑ The Author(s). 2020 Open Access This article is licensed under a Creative Commons Attribution 4.0 International License which permits use, sharing, adaptation, distribution and reproduction in any medium or format, as long as you give appropriate credit to the original author(s) and the source, provide a link to the Creative Commons licence, and indicate if changes were made. The images or other third party material in this article are included in the article's Creative Commons licence, unless indicated otherwise in a credit line to the material. If material is not included in the article's Creative Commons licence and your intended use is not permitted by statutory regulation or exceeds the permitted use, you will need to obtain permission directly from the copyright holder. To view a copy of this licence, visit http://creativecommons.org/licenses/by/4.0/. 
maintenance of structures. Advanced non-destructive testing and evaluation (NDT \& E) techniques appear to be great solutions to address these deteriorated bridges, as they can facilitate fast, cost-effective, efficient, rapid data collection, and reliable condition assessment of existing structures. These techniques are also becoming increasingly important given the facts that they can be carried out without significant detriment to bridge structures and potentially without the need for traffic interruptions $[6,22]$. This study aims at utilizing the NDT \& E method to assess current conditions of concrete bridge components such as reinforced-concrete slabs.

Imaging of concrete structures can be performed by one or combinations of the following major NDT \& E techniques: electromagnetic based (e.g., ground penetrating radar, infrared thermography, and radiography) and stress-wave based (e.g., impact echo and ultrasonic pulse velocity). Each NDT \& E technique has its own advantages and limitations [1]. For example, ground penetrating radar (GPR) with high-frequency antenna (usually higher than $900 \mathrm{MHz}$ ) can obtain strong signal reflections from embedded objects (a.k.a. inclusions) in concrete structures. The thermographic testing method can generate a rapid mapping of large surface areas, but it is susceptible to temperature fluctuations and requires a highly uniform heat source.

The ultrasonic technique (a.k.a. acoustic-based method) is the most applied imaging methods in medicine and has been used successfully for many years to evaluate metallic and composite materials $[15,23]$. First tests on concrete specimens using the ultrasonic (UT) methods began in the early 1990s. Presently, major practical applications of the UT methods for concrete structures include: (1) To measure the compressive strength, elastic modulus, and thickness [14, 21] (2) To locate rebars and tendon ducts ([20]); and (3) To detect inclusions in concrete structures $[15,24,25]$. According to [22], the acoustic-based UT shear-wave tomography technique can provide $2 \mathrm{D}$ and/or $3 \mathrm{D}$ visualizations of concrete structures with ease, speed, and efficiency and it would be preferred for imaging concrete structures.

Two-sided UT testing methods require the coupling of transmitters and receivers in two sides of structures with couplants and gels, which may not be applicable in the field because of limitations in physical coupling and surface preparation [23]. Further, the UT wave is attenuated when propagating through a large concrete structure, and small inclusions cannot be captured [7]. One-sided UT array technology can overcome the shortcoming of the two-sided UT techniques. Several techniques were developed, such as the phased array ultrasonic testing (PAUT) and the UT pitch-catch method. The main differences between the two methods are transducer configurations and field applications as discussed in the next paragraphs.

Phased array technology uses a matrix of multiple single transducers (up to 128 transducers) into one probe and electronic time delays to create UT beams that can be steered, scanned, swept, and focused electronically for fast inspection, full data storage, and multiple angle inspections [9]. The most common application of PAUT is weld inspections [3], and a generic examination would be looking for flaws oriented parallel to the weld centreline [9]. In addition, PAUT also applied for mapping corrosion for steel bridges and ancillary structures [17]. Limited applications of PAUT method for concrete structures were found in the literature [18].

The UT pitch-catch method has been developed to detect inclusions in full-scale reinforced concrete structures [7]. The dry-point-contact transducer array unit is available to overcome the UT contact problems (i.e., difficulty of preparing the surface to the required condition), make it possible to test the concrete structures with minimal surface preparations. The commerciallyavailable UPC device (Fig. 1) is a low-frequency ultrasonic shear-wave tomography device that can be used to image the subsurface concrete condition in a matter of few seconds. The measurement unit of the UPC device composes of 48 dry-point-contact (DPC) transmitting and receiving transducers (with ceramic wear-resistant tips) arranged in a matrix antenna array with 12 channels (channels $C_{1}$ through $C_{12}$ as shown in Fig. 1b) at a longitudinal spacing of $30 \mathrm{~mm}$; each channel is composed of four transducers at a transverse spacing of 25 $\mathrm{mm}$ (Fig. 1b). The use of the DPC transducers allows inspection/testing on a rough and uneven surface without surface preparation [19]. Besides, couplant, a liquid material that uses to facilitate the transmission of ultrasonic energy from the transducer into the test object, is not required for this UPC device. This is due to the fact that the DPC transducers can overcome the large acoustic impedance mismatch between air and solids (i.e., the test object).

Figure 2 shows the basic principles of the UPC device. It is based on the ultrasonic pulse-echo method using transmitting and receiving transducers in a "pitch-catch" configuration (Fig. 2a). In the pitch-catch method, one channel $C_{\mathrm{i}}(i \in[1: 11])$ acting as a transmitter emits shear-wave pulse into the test object, and the other channels $C_{j}(j \in[(i+1): 12])$ sequentially act as a receiver picking up direct and reflected pulse (a.k.a. echo) [16]. As a result, the 1st channel only acts as a transmitter while the 12th channel always serves as a receiver. For example, Fig. 2b shows the first channel acting as the transmitter and other channels acting as receivers while Fig. 2c exhibits the second channel as the transmitter and other channels (except the first and second 


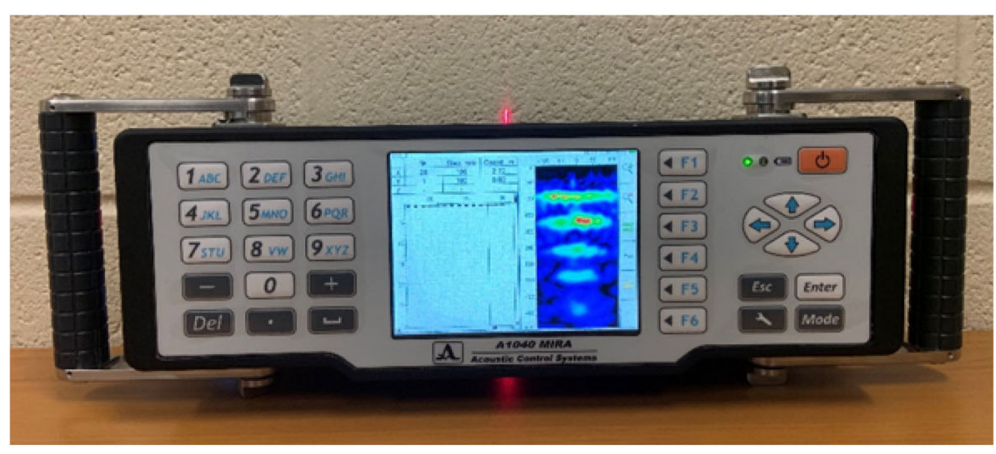

(a) Front panel of the UPC device

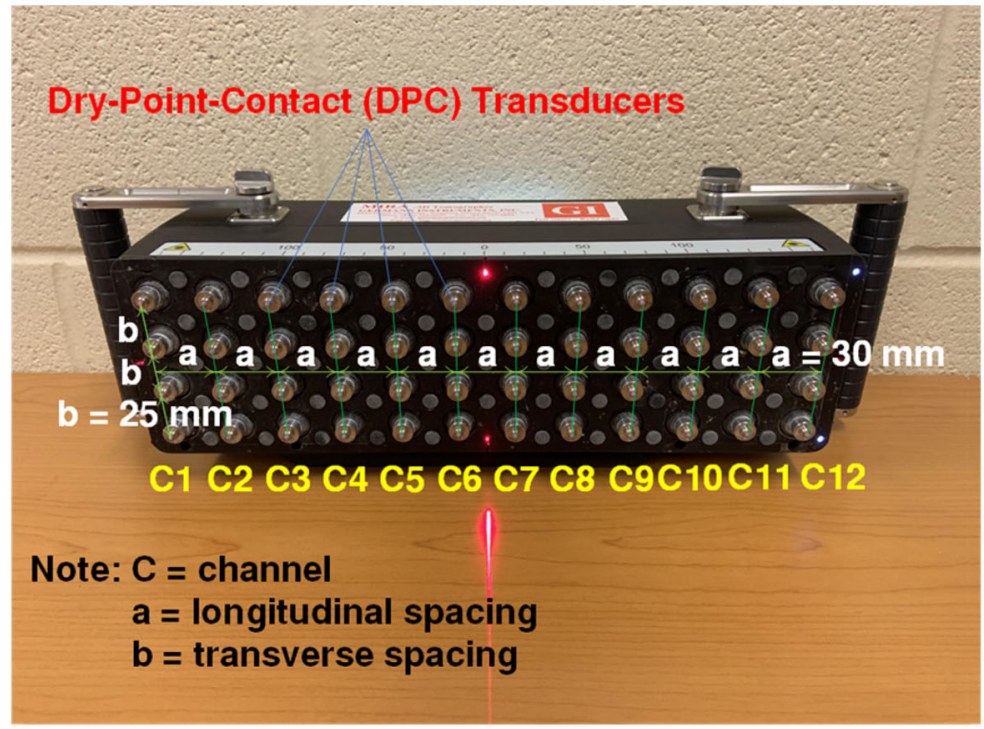

(b) 48 low-frequency DPC transducers

Fig. 1 Commercially-available UPC device. a Front panel of the UPC device. b 48 low-frequency DPC transducers

channels) as receivers. Based on this principle, there are a total of 66 transmitting-receiving pairs or 66 data arrays for each B-scan. The depth of the reflecting interface (i.e., defects, anomalies, discontinuities, irregularities, etc.), $Z$, can be calculated by Eq. 1 .

$$
Z=\sqrt{\left(C_{s} \frac{\Delta t}{2}\right)^{2}-X^{2}}
$$

where $C_{s}=$ shear-wave speed; $\Delta t=$ measured travel time; $X=$ horizontal spacing between the first channel and the test location (Fig. 2a).

Synthetic aperture focusing technique (SAFT), a digital signal processing method for ultrasonic testing, is used to reconstruct 2-D images of the interior of the test object (i.e., the RC slab in this study) at the test location. SAFT was originally developed in the early 1970s based on synthetic aperture airborne radar systems [10]. Its algorithm numerically superimposes pulse-echo signals measured at several positions, thus structural noise is suppressed by spatial superposition and resulting in a high-resolution image [25].

Hoegh et al. [12] investigate assurance/quality control of concrete pavements using the UPC device. Results of multiple field trials indicated that the UPC device is capable of determining concrete pavement thickness and locations of reinforcements. The results also showed that this device is adequate to detect flaws such as delamination and degradation at pavement joints. Although the commercially-available UPC device comes with data interpretation proprietary software (that uses the synthetic aperture focusing technique), it is necessary to emphasize the importance of developing an automated data interpretation process for broader applications. This 


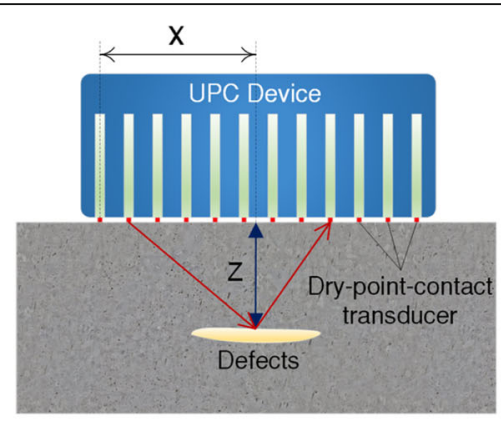

(a) Transmitting and receiving transducers in a "pitch-catch" configuration

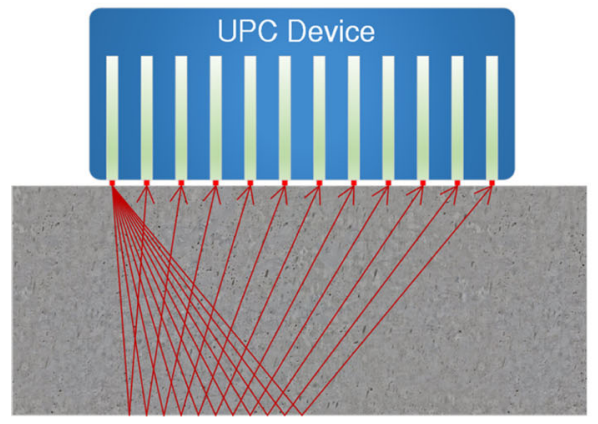

(b) The first channel of transducers

transmitting signals and subsequently receiving by other channels



(c) The second channel of transducers

transmitting signals and subsequently receiving by other channels

Fig. 2 Basic principles of the UPC device. a Transmitting and receiving transducers in a "pitch-catch" configuration. b The first channel of transducers transmitting signals and subsequently receiving by other channels. $\mathbf{c}$ The second channel of transducers transmitting signals and subsequently receiving by other channels

automation process can eliminate the need for specialized and experienced/expertise users and result in the productivity of data interpretation.

Bishko et al. [5] present field applications of the UPC device for concrete testing. Advanced methods of computer tomography and SAFT algorithm were used for signal processing. The authors concluded that the UPC device can be applied for detection and size/location estimations of different objects inside concrete such as ducts, rebars, delaminations, air hollows, etc. Although the SAFT algorithm employed in the UPC device's proprietary imaging software is based on a modified SAFT (known as SAFT-Combinations or SAFT-C) to enhance the signal-to-noise ratio and image resolution [2], it has limited the application of the UPC device because single-layer homogeneous medium (e.g., concrete) and constant shear-wave velocity have been assumed. In practice, bridge components such as bridge decks include multiple-layers of overlays of different materials with varying shear-wave velocities. As a result, the proprietary software may underestimate or overestimate information on the multi-layer bridge deck (e.g., depth, thickness, internal defects) [16]. Also, the Hilbert transform used in the signal processing by the proprietary software may result in an inaccurate presentation of reflected waves at the backwalls or discontinuities (i.e., misleading information on the tested components). This study aims at developing inhouse software with a modified SAFT algorithm (more effective than the original SAFT and the afore-mentioned SAFT-C algorithms) and proposes a novel signal filtering method for accurate predictions (e.g., locations and dimensions) of internal defects of reinforced concrete slabs.

\section{Developed software}

Computer code with a fully graphical interface (written in Borland Delphi) was developed to overcome the shortcoming of the current commercial UPC device's proprietary software for ultrasonic imaging of concrete structures. The most significant advantages of the inhouse developed software include 1) developed a new procedure to calculate the approximate shear-wave velocity in a media using direct arrival time from each measurement pairs; 2) produced a single panoramic reconstruction from multiple overlapping scans by using a superposition algorithm; 3) proposed novel signal 
filtering and processing (i.e., sharp and high-resolution reconstructed images of the embedded objects are obtained with the images synthesized from the ultrasonic linear array); 4) determined the embedded object in the $\mathrm{RC}$ structure from signal amplitudes obtained on vertical and horizontal sections of the $2 \mathrm{D}$ reconstructed image that reveals information on dimensions and exact locations of the embedded objects.

\section{Modified SAFT (synthetic aperture focusing technique)}

In the ultrasonic pitch and catch (a.k.a. pulse-echo) method, travel time from transmitter to receiver and amplitude of pulses reflected at an object embedded in the concrete structures are measured. Synthetic apertures focus the signal amplitude to any point in the sampling area of large transducers by coherent superposition. Initially, the synthetic aperture focusing technique (SAFT) is developed to interpret signals with transmitting and receiving at the same location. This technique also can be applied for an array of transmitters and receivers located at different locations. Twodimensional (2D) SAFT is obtained from the linear aperture and represented by a B-scan cross-section. A threedimensional (3D) SAFT is synthesized from the planar synthetic apertures of two perpendicular directions, such as B-scan and A-scan.

In this study, the original SAFT is modified and improved to interpret signals from transmitting and receiving transducers located at different locations on one side of the concrete component. In an ultrasonic linear array of transmitting and receiving pair, the impulse respect to a travel time represents response characteristics of positions in the media that have the same travel-path length. Synthesizing the overall signal produces a reconstructed

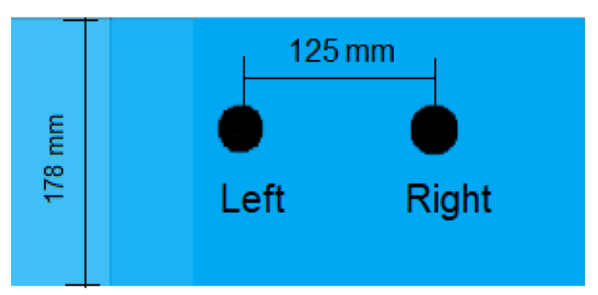

a) RC slab and embedded rebars

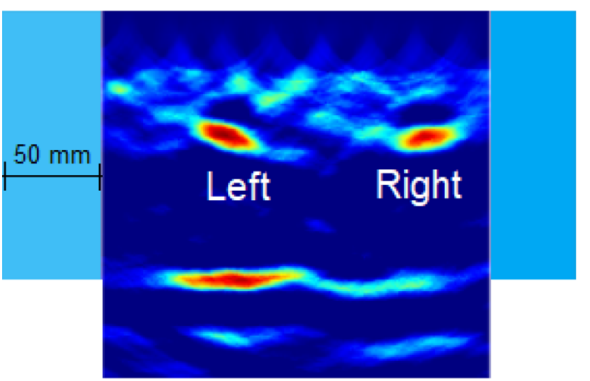

c) The second scan

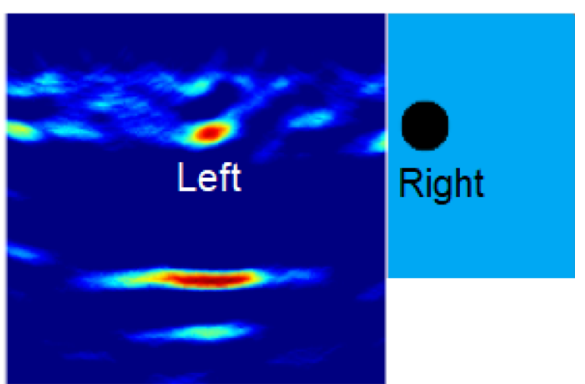

b) The first scan

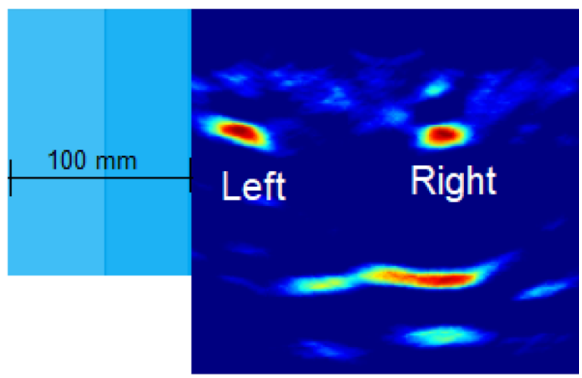

d) The third scan

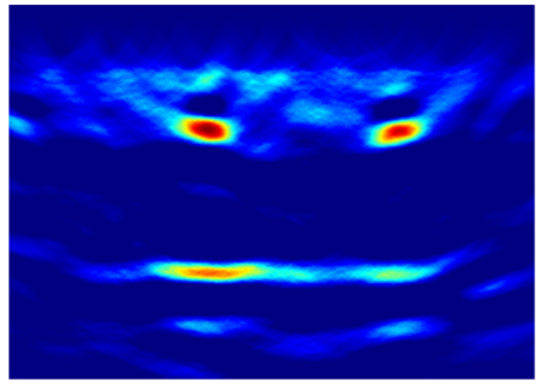

e) Combined overlapping scan

Fig. 3 Three overlapping B-scans and their combination. a RC slab and embedded rebars. $\mathbf{b}$ The first scan. $\mathbf{c}$ The second scan. $\mathbf{d}$ The third scan. e Combined overlapping scan 
image referred to as a SAFT B-scan. The relationships between the synthesized signal and the ultrasonic linear array are given in the following equations:

$$
\begin{gathered}
g_{i j}=\sum_{t=1}^{N-1} \sum_{r=t+1}^{N} A\left(x_{t}, x_{e}, x_{i}, z_{k}\right) \phi_{t r}\left(x_{i}, z_{j}\right) \\
\phi_{t r}\left(x_{i}, z_{j}\right)=s\left(x_{t}, x_{r}, \frac{1}{c}\left(\sqrt{\left(x_{i}-x_{t}\right)^{2}+z_{j}^{2}}+\sqrt{\left(x_{i}-x_{r}\right)^{2}+z_{j}^{2}}\right)\right)
\end{gathered}
$$

where $g_{i j}$ is synthesized impulse at each position within the region of interest (ROI); $i$ and $j$ are horizontal and vertical indexes of a position in the ROI; $N$ is number of transducer channels; $t$ and $r$ are indexes of the transmitting and receiving transducers; $x_{t}$ and $x_{r}$ are horizontal coordinates of the transmitting and receiving transducers; $x_{i}$ and $z_{j}$ are horizontal and vertical coordinates of a position in the ROI with the $i^{\text {th }}$ and $j^{\text {th }}$ indexes; $\phi_{t r}$ and $s$ is a function of the magnitude of the impulse at an evaluated time; and $A$ is the apodization factor expressed as follows:

$$
\begin{aligned}
& A\left(x_{t}, x_{e}, x_{i}, z_{k}\right)=\alpha_{t}\left(x_{t}, x_{i}, z_{k}\right) \alpha_{r}\left(x_{r}, x_{i}, z_{k}\right) \\
& \alpha_{t}\left(x_{t}, x_{i}, z_{k}\right)=\frac{z_{j}}{\sqrt{\left(x_{i}-x_{t}\right)^{2}+z_{j}^{2}}} \\
& \alpha_{r}\left(x_{r}, x_{i}, z_{k}\right)=\frac{z_{j}}{\sqrt{\left(x_{i}-x_{r}\right)^{2}+z_{j}^{2}}}
\end{aligned}
$$

where: $\alpha_{t}$ and $\alpha_{r}$ are cosines of the angles between travel paths and vertical lines at the locations of transmitting

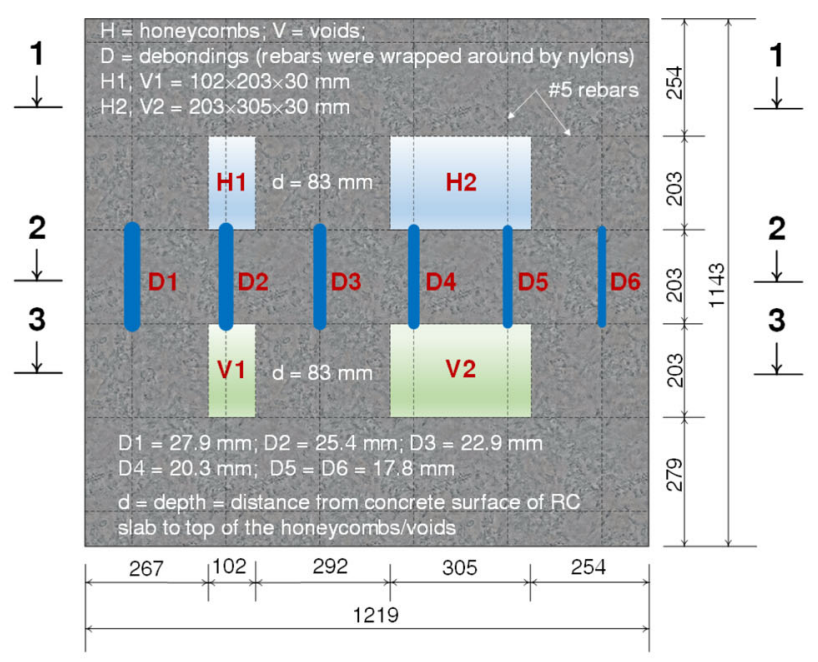

(a) Locations of artificial voids, debondings, and honeycombs

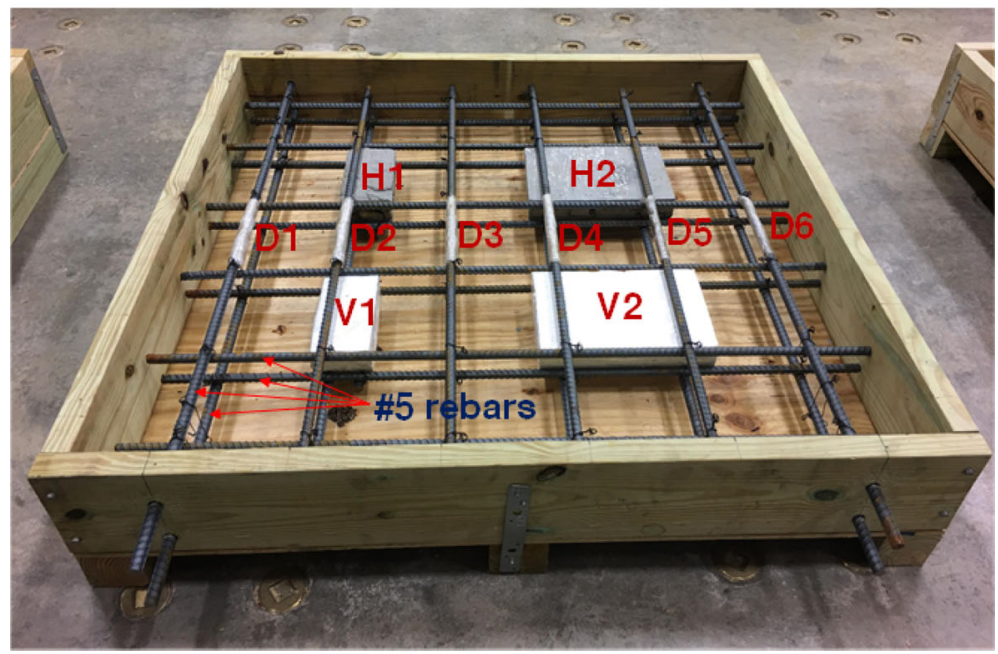

(b) Schematic view

Fig. 4 Details of the RC slab specimen. a Locations of artificial voids, debondings, and honeycombs. b Schematic view 
and receiving transducers, respectively. The above equations allow for eliminating the direct arrival impulse (i.e., reduction of the impulse near the surface, where $z_{j}$ is negligible).

To cover for a large ROI, multiple overlapping scans are combined into a single panoramic reconstructed image. The scans are conducted at equal spacings in the direction along the aperture of the linear array. In this case, all impulses from the individual scan are accumulated according to their physical locations. The amplitude of the impulse, $G_{i j}$, is determined from the individual impulse as the following equation:

$$
G_{i j}=\sum_{m=1}^{M} g_{i j}^{m}
$$

where $M$ is the number of overlapping scans; $m$ is the index of a scan.

The Hilbert transform focuses the instantaneous amplitude within the ROI to change in acoustic impedance that can obtain high reflectivity in reconstruction for the location of the embedded objects. However, it cannot distinguish rebars and voids when they appear in the same form in the reconstructed image. Therefore, in this study, the Hilbert transform is dismissed for detecting not only the location but also the characteristics of embedded objects in the RC structure.

Figure 3 demonstrates the SAFT panoramic algorithms, which three overlapping B-scans of two rebars (embedded in a $178 \mathrm{~mm}$ thick concrete slab and with $125 \mathrm{~mm}$ spacing) are combined. The scans were conducted from left to right with a spacing of $50 \mathrm{~mm}$, as shown in Fig. 3b-d. The overlapping scans were then combined using Eq. 7 and the resulting reconstructed image is presented in Fig. 3e. All scans show highintensity reflections (dark-red color) at the rebar locations and slab bottom (i.e., backwall reflection). This example proved that the proposed algorithm effectively reconstructs the visualization image from multiple overlapping scans.

\section{Experimental program Specimen preparation and details}

To validate the effectiveness of the inhouse developed software, a reinforced concrete (RC) slab was designed with two layers of \#5 steel rebars and artificial defects (Fig. 4). Overall dimensions of the RC slab were $114.3 \times$ $121.9 \times 17.8 \mathrm{~cm}$ (width $\times$ length $\times$ thickness). The artificial defects embedded in the RC slab included honeycombs, voids, and debondings. The honeycombs were made of cobbles and low-grade mortar. Expanded polystyrene (EPS) foam was used to represent the artificial voids as it had the same dielectric properties as air. Two sizes of honeycombs and voids were prepared: small and large sizes with dimensions of $102 \times 203 \times 30 \mathrm{~mm}$ and $305 \times 203 \times 30 \mathrm{~mm}$ (width $\times$ length $\times$ thickness), respectively. The rebars were wrapped around by thin plastic

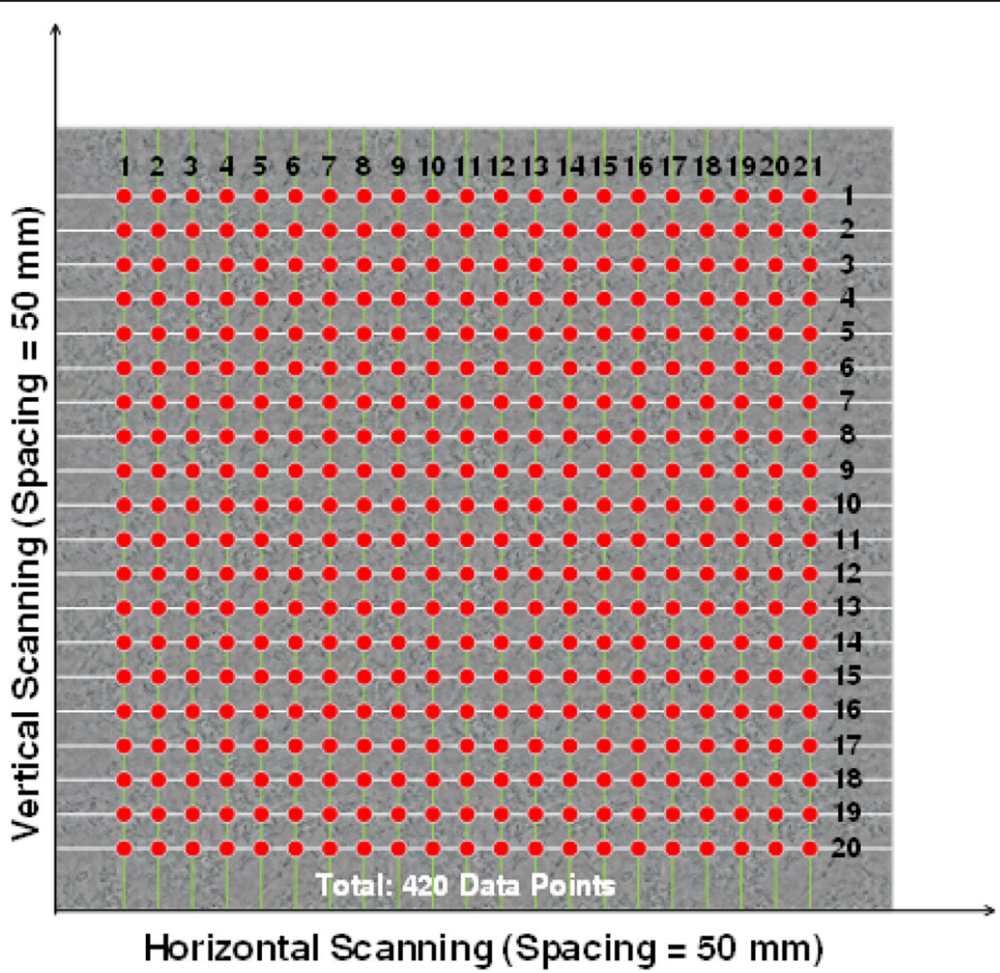

Fig. 5 Data collection on the RC slab using the UPC device 
Table 1 Key parameters of the UPC Device [19]

\begin{tabular}{|c|c|c|c|}
\hline Parameter & Unit & Value & Description \\
\hline Color gain & $\mathrm{dB}$ & 10 & The brightness of the reconstructed B-scan image \\
\hline Analog gain & $d B$ & 20 & Amplification applied to the receiving transducer signals \\
\hline $\begin{array}{l}\text { Operating } \\
\text { frequency }\end{array}$ & $\mathrm{kHz}$ & 50 & $\begin{array}{l}\text { Center frequency of transmitted pulse; varied between } 25 \text { to } 85 \mathrm{kHz} \text { depending on material and depth of test object; } \\
50 \mathrm{kHz} \text { is recommended as default value }\end{array}$ \\
\hline Velocity & $\mathrm{m} / \mathrm{s}$ & 2450 & Manually entered shear-wave speed for use in SAFT reconstruction \\
\hline Delay & $\mu S$ & 22 & Time for pulse to travel through the device \\
\hline Image quality & - & High & From low to high \\
\hline Horizontal step & $\mathrm{mm}$ & 50 & Horizontal spacing of grid points \\
\hline Vertical step & $\mathrm{mm}$ & 50 & Vertical spacing of grid points \\
\hline
\end{tabular}

$d B$ decibel

film to represent artificial debondings of the rebars. The diameters of the plastic-wrapped rebars were varied between $17.8 \mathrm{~mm}$ and $27.9 \mathrm{~mm}$ (Fig. 4a). Ready-mix concrete from in-transit mixers was used for the casting of the RC slab. The slab specimen was then cured and tested in laboratory conditions.

\section{Data collection and acquisition}

The data collection was performed in both horizontal and vertical directions with equal spacing of $50 \mathrm{~mm}$ (Fig. 5). A total of 420 data points (corresponding to the grid point locations in Fig. 5) were collected on the validation RC slab. The operating frequency was set to a default value of $50 \mathrm{kHz}$ while the shear-wave velocity was set to be determined by actual measurements. By turning on the "using the measured velocity" option, the time for the pulse travel through the device (a.k.a. "delay" time) is automatically measured (in microseconds). Table 1 lists the key parameters of the device used for the data collection. Detailed descriptions are provided for each parameter. It should be noted that the shear-wave pulse velocity of $2450 \mathrm{~m} / \mathrm{s}$ was assumed for concrete and this value was used in SAFT reconstruction by the device.

\section{Results and discussions}

The signal amplitudes for the reconstructed image of the concrete slab obtained from SAFT without applying the Hilbert transform can be positive and negative values and mapped in a color range from black (minimum negative to zero) to dark red (maximum positive), as shown in Fig. 6. The color spectrum is defined in Fig. 6 with "dark" designates black to blue colors and "bright" indicates light-blue to dark-red colors. Figure 7 exhibits the 2D B-scan reconstructed images using the in-house developed software for the detection of rebars, artificial debondings, and voids embedded in the RC slab.

The bottom of the concrete slab (a.k.a. backwall reflection) has a clear polarized bright-dark pattern with a bright-color strip on top and dark-color strip underneath, as shown in Fig. 7. The backwall reflection can also be determined from the positive-negative waveform pattern (Fig. 7b). The thickness of the concrete slab can be approximated by the depth of the positive peak of the signal amplitude. The calculated thickness of the concrete slab (based on the reconstructed images) ranges from $182 \mathrm{~mm}$ to $191 \mathrm{~mm}$, which is approximately $2.2 \%-7.3 \%$ higher than the design thickness of the slab. These discrepancies may be caused by the uneven surface of the RC slabs. Similar observations were reported by other researchers $[8,23]$.

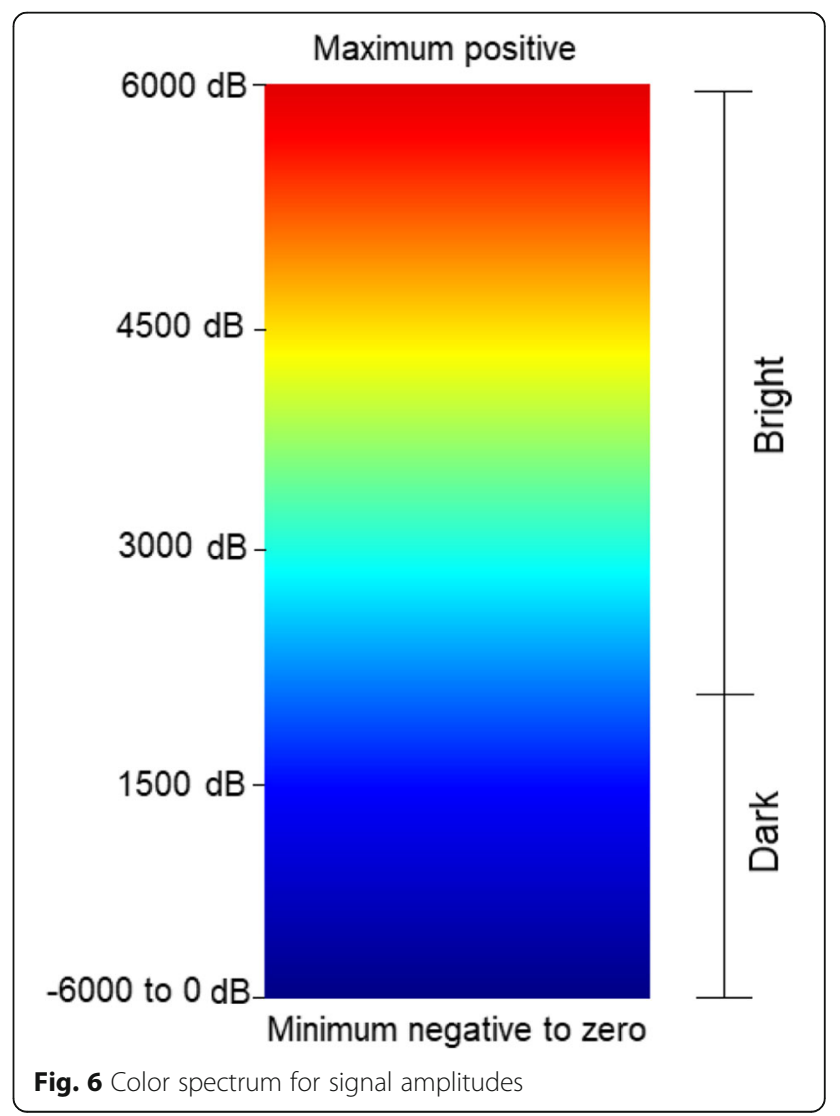




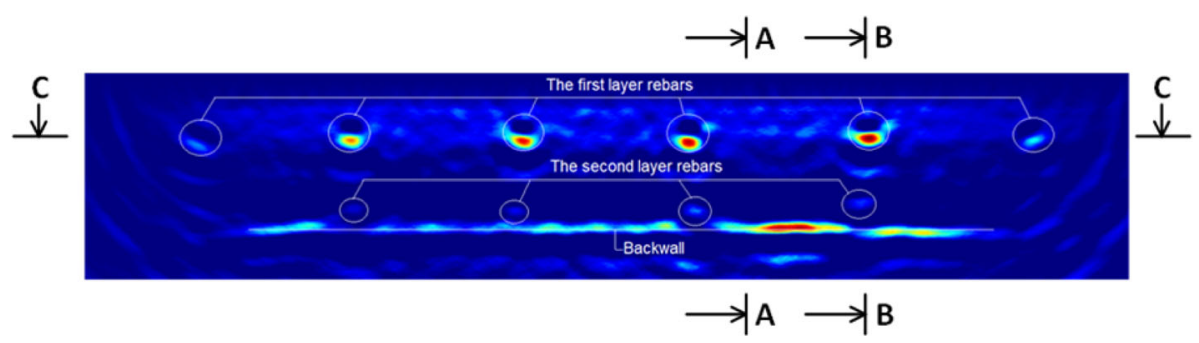

a) $2 \mathrm{D}$ reconstructed image at the location of the fully bonded rebars (refer to section 1-1 in Fig.

4a)

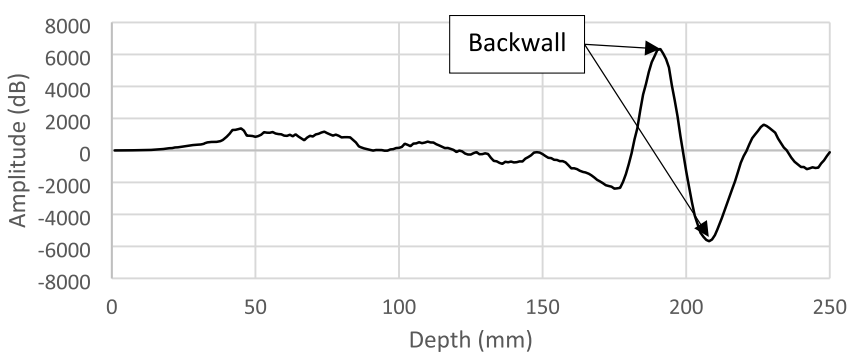

b) Backwall reflection at section A-A (refer to Fig. 7a)

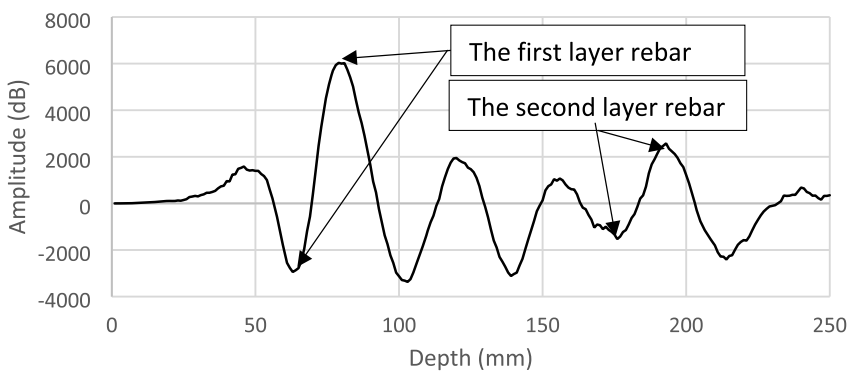

c) Amplitude versus depth relationship at the location of a fully-bonded rebar (refer to section B-

B in Fig. 7a)

Fig. $72 \mathrm{D}$ reconstructed image of backwall and fully-bonded rebars. a 2D reconstructed image at the location of the fully bonded rebars (refer to section 1-1 in Fig. 4a). b Backwall reflection at section A-A (refer to Fig. 7a). c Amplitude versus depth relationship at the location of a fullybonded rebar (refer to section B-B in Fig. 7a)

In Fig. 7a, the first layer of the fully bonded rebars (i.e., rebars without the thin plastic wraps) are highly visible in the reconstructed image (i.e., areas with bright color and dark shadows above). Locations of rebars can be determined not only from the reconstructed images but also from the signal amplitudes either in vertical or horizontal directions. In the vertical direction (Fig. 7c), the rebar is represented by a waveform with a minimum peak followed by a maximum peak. This waveform usually appears when the ultrasonic wave reflects from higher density inclusions such as rebars embedded in the concrete. The depth of the rebar is equal to the mean value of those two-peak coordinates that can provide an excellent approximation to the measured depth. The rebar locations in the horizontal direction are accurately identified based on peak amplitudes of the waveform (refer to circular marks on the horizontal section of the 2D reconstructed image as shown in Fig. 8). The rebars in the second layer are not obviously observed in the reconstructed image because of the signal lost due to reflection from the first layer and attenuation (Fig. 7a). However, these rebars have a similar waveform pattern as the rebars in the first layer. Locations of the second-layer rebars can be determined based on the 


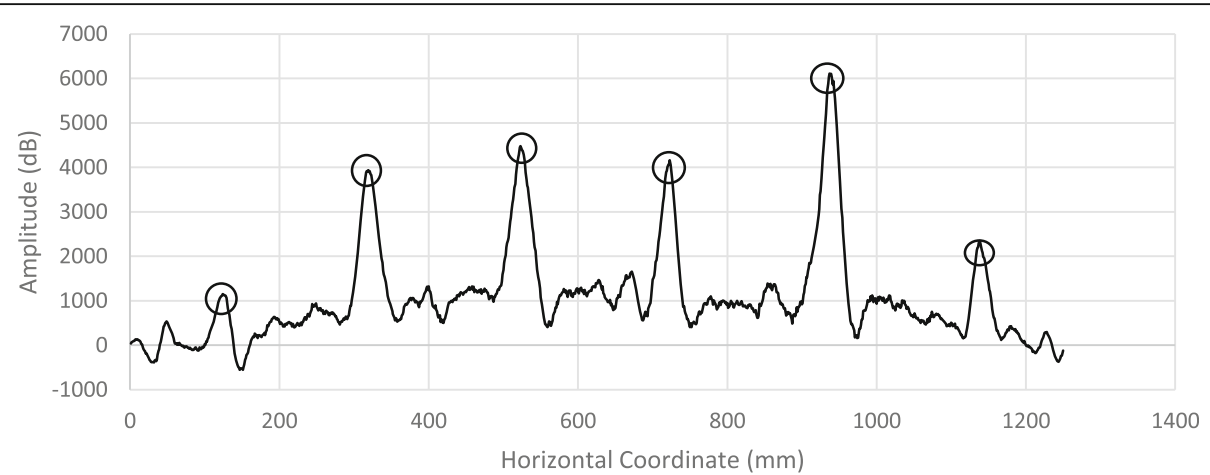

Fig. 8 Amplitude versus horizontal coordinate relationship at the location of the first-layer rebars (refer to section C-C in Fig. 7a)

waveform pattern, although the peaks of the signal amplitudes of the second-layer rebars are lower than those of the first-layer rebars (Fig. 7c). The rebars near the edges of the concrete slab appear in different sizes and shapes in comparison with the interior rebars. It can be observed that those rebars are located in areas with fewer scans causing limited aperture effects [11]. Finer scan spacings near the edges of the RC structure is thus recommended to overcome the reconstructed $2 \mathrm{D}$ images.
The artificial debonded rebars (i.e., rebars wrapped with thin plastic films of varying thicknesses, as shown in Fig. 4) are detected with absolutely different patterns in comparison to those of the fully-bonded rebars. The reflection amplitude from a debonded rebar has a sequential form of bright-dark-bright pattern (Fig. 9a) or positive-negative-positive waveform (Fig. 9b) in the vertical direction. This phenomenon occurs when the ultrasonic shear wave reflects from an object embedded in the concrete slab with a higher density wrapped by a

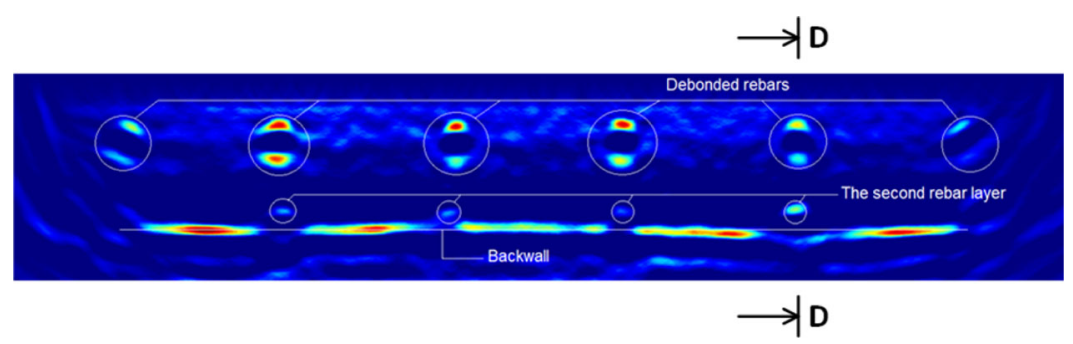

a) $2 \mathrm{D}$ reconstructed image at the location of the artificial debonded rebars (refer to section 2-2 in

Fig. 4a)



b) Amplitude versus depth relationship at the location of an artificial debonded rebar (refer to section D-D in Fig. 9a)

Fig. $92 \mathrm{D}$ reconstructed image of artificial debonded rebars. a 2D reconstructed image at the location of the artificial debonded rebars (refer to section 2-2 in Fig. 4a). b Amplitude versus depth relationship at the location of an artificial debonded rebar (refer to section D-D in Fig. 9a) 
thin layer of lower density than concrete material that helps to identify the debonded rebars. The location of the debonded rebar is likely at the center of the dark spot in the bright-dark-bright pattern area (Fig. 9a) or negative amplitude region (Fig. 9b). The reflected waves of the debonded rebars are in contrast with the fullybonded rebars. This result implies that the thickness of the plastic-wrapped layer of the debonded rebars has effects on the reflected wave. It is observed that the thickness of the debonded rebars is decreasing from left to right based on the size of dark spots in the brightdark-bright pattern areas (Fig. 9a).

Figure 10a shows the reconstructed image obtained from the developed software with a precise indication of the locations and horizontal dimensions of the artificial voids. As discussed earlier, when the ultrasonic shear

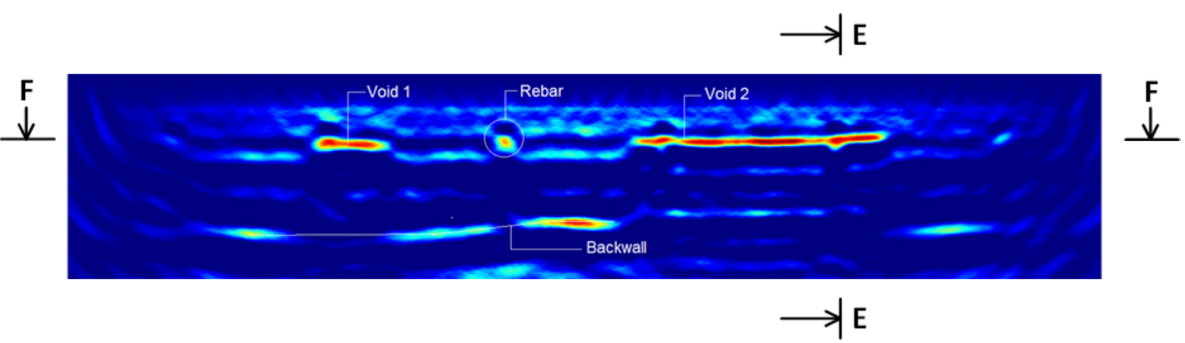

a) 2D reconstructed image at the location of the artificial voids (refer to section 3-3 in Fig. 4a)



b) Amplitude versus depth relationship at the location of an artificial void (refer to section E-E in

Fig. 9a)

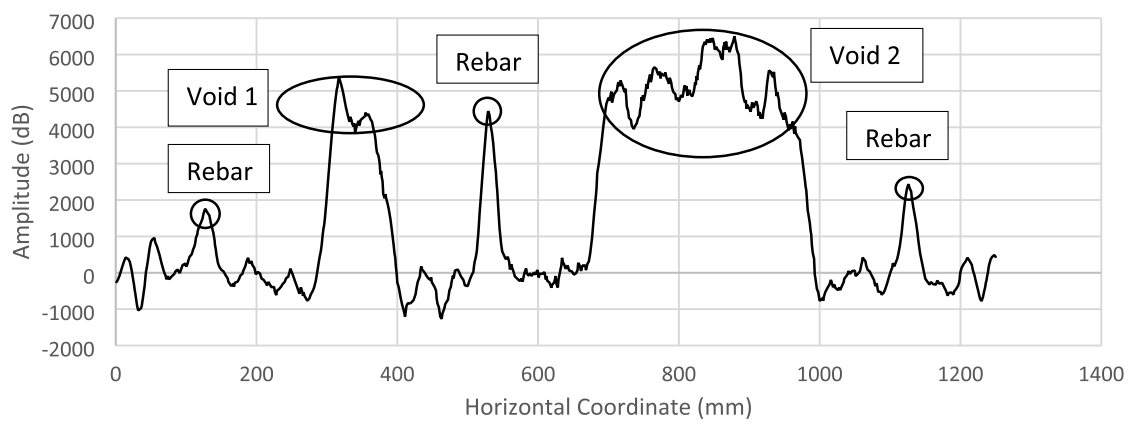

c) Amplitude versus horizontal coordinate relationship at the location of artificial voids and

fully-bonded rebars (refer to section F-F in Fig. 9a)

Fig. $102 \mathrm{D}$ reconstructed image of artificial voids. a 2D reconstructed image at the location of the artificial voids (refer to section 3-3 in Fig. 4a). b Amplitude versus depth relationship at the location of an artificial void (refer to section E-E in Fig. 9a). c Amplitude versus horizontal coordinate relationship at the location of artificial voids and fully-bonded rebars (refer to section F-F in Fig. 9a) 
wave reflects from a lower density material, the waveform has a dark-bright pattern or positive-negative peak sequence in the vertical direction (Fig. 10b) as a recognizable sign of the void. Horizontal dimensions of the void 1 (V1) and void 2 (V2) was $113 \mathrm{~mm}$ and 325 $\mathrm{mm}$, respectively, which was determined based on the maximum breadth of the positive signal amplitude area (Fig. 10c). For all artificial voids, the maximum discrepancy between the prediction and the actual measurement (Fig. 4a) of the horizontal dimensions is approximately $6.5 \%$.

The honeycombs, which were made of cobbles and lowgrade mortar, could not be detected by the UPC device. This may be attributed to the fact that the simulated cavities in the honeycombs are too small and/or the densities of the honeycombs are similar to the concrete material. As a result, the honeycombs are not visible as the signal amplitudes of the honeycombs are almost comparable to the amplitudes of the surrounding concrete.
Figures 11 shows 2D images obtained from the UPC device's proprietary software for the fully-bonded rebars and artificial debonded rebars \& voids. In general, the software provides fairly good visualization of the embedded objects in terms of their locations and horizontal dimensions. However, by applying the Hilbert transform, the images of the embedded objects are much bigger than their actual measured dimensions in the vertical direction. Additionally, the proprietary software cannot recognize the fully-bonded rebars as well as the debonded rebars since they have a comparable color spectrum, as shown in Fig. 11a-b.

\section{Conclusions and future works}

This study presents a methodology based on an improved ultrasonic synthetic aperture focusing technique (SAFT) to reconstruct images of reinforced-concrete slabs. Computer software with a fully graphical interface was developed to overcome the shortcoming of the current

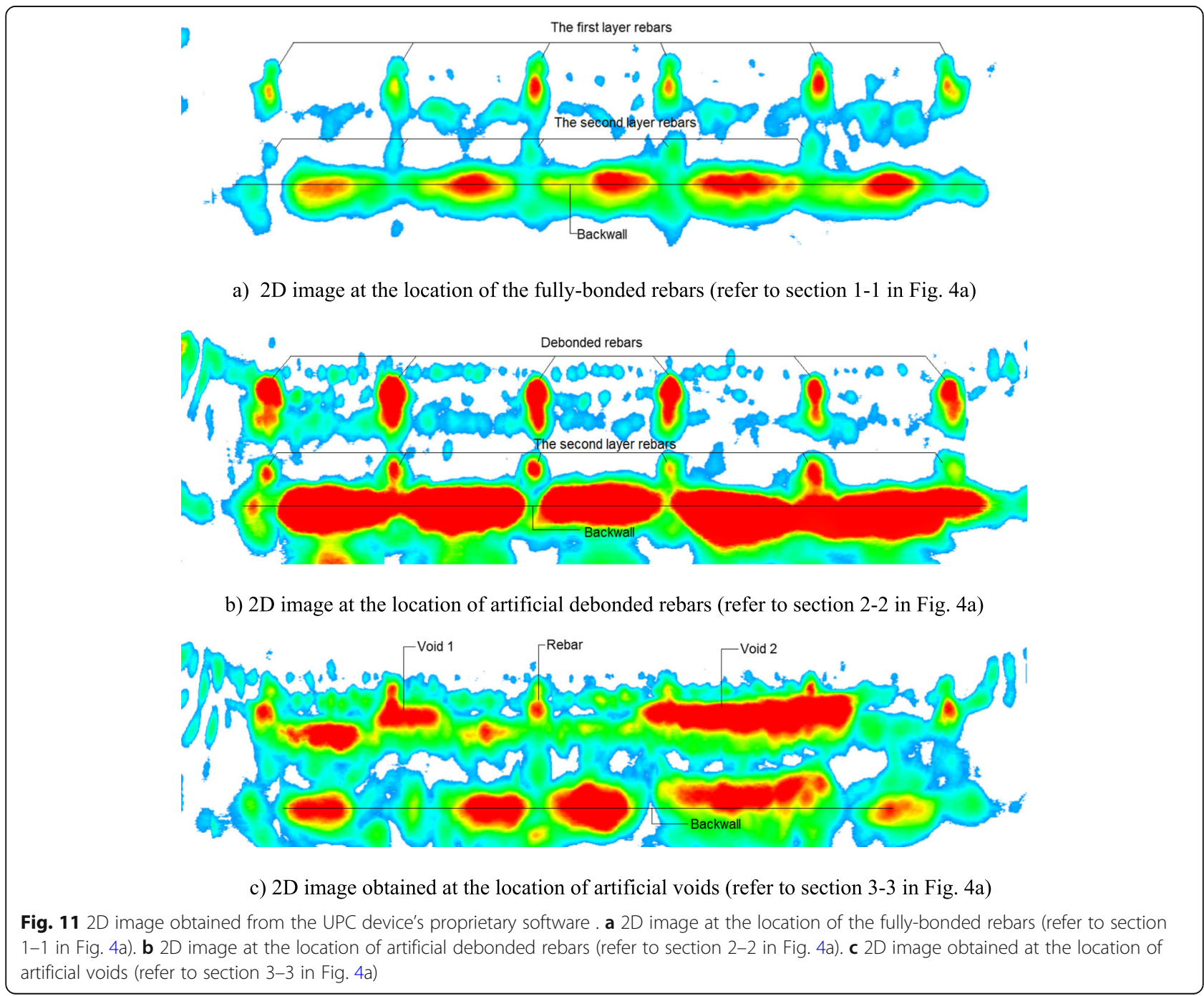


commercial ultrasonic pitch and catch (UPC) device's proprietary software for ultrasonic imaging of concrete structures. The developed software was effectively visualized the embedded objects in the concrete slab including fullybonded rebars, and artificial debonded rebars and voids. The results reveal that rebars and different types of defects can be interpreted based on specific patterns of the color spectrum and waveform in the vertical direction. The fully-bonded rebar is represented by a waveform with a minimum peak followed by a maximum peak, while waveforms of the artificial voids are reversed. The proposed method reveals that the reflection amplitude from a debonded rebar has a sequential form of bright-darkbright pattern or positive-negative-positive waveform.

The UPC is proved to be an excellent non-destructive testing technique for the accurate evaluation of concrete structures. However, the data collection process is usually labor-intensive and time-consuming (depending on the finest of the scanning grids as well as the tested/survey areas). Our future study will focus on developing a climbing robot to automate the data collection process. The developed software will also be improved and extended to provide real-time visualization of concrete structures. With the developed software and the automated data collection, the inspection of constructed facilities can be facilitated.

\section{Abbreviations \\ 2D: Two Dimensional; 3D: Three Dimensional; ACl: American Concrete Institute; ASCE: American Society of Civil Engineers; DPC: Dry Point Contact; EPS: Expanded Polystyrene; NDT: Non-Destructive Testing; NDT \& E: Non-Destructive Testing and Evaluation; PAUT: Phased Array Ultrasonic Testing; RC: Reinforced Concrete; ROI: Region of Interest; SAFT: Synthetic Aperture Focusing Technique; SAFT- C: SAFT-Combinations; UPC: Ultrasonic Pitch and Catch}

\section{Acknowledgments}

The authors would like to thank Dr. Tu Nguyen and Dr. Kien Dinh for their assistances in this research project.

\section{Authors' contributions}

WAZ, HDN, and HMN helped shape the research, analysis, and manuscript. WAZ and HDN supervised the entire project. HDN and HMN conceived of the presented ideas and wrote the manuscript. HDN proposed the experimental program and conducted laboratory tests. HMN developed visualization software and analysis. All authors read and approved the final manuscript.

\section{Funding}

Not applicable.

\section{Availability of data and materials}

All data generated or analyzed during this study are included in this published article.

\section{Competing interests}

The authors declare that they have no competing interests.

Received: 22 April 2020 Accepted: 14 September 2020

Published online: 07 October 2020

\section{References}

1. ACl Committee 228 (2013) Report on nondestructive test methods for evaluation of concrete in structures. Report ACl 228.2R-13. American Concrete Institute, Farmington Hills
2. Aldo O, Samokrutov AA, Samokrutov PA (2013) Assessment of concrete structures using the Mira and Eyecon ultrasonic shear wave devices and the SAFT-C image reconstruction technique. Constr Build Mater 38:1276-1291

3. Anandamurugan S (2009) Manual phased array ultrasonic technique for weld application. In: Proceedings of the national seminar \& exhibition on non-destructive evaluation, NDE 2009

4. ASCE (2017) 2017 infrastructure report card. American Society of Civil Engineers (ASCE), Reston

5. Bishko A, Samokrutov AA, Shevaldykin VG (2008) Ultrasonic echo-pulse tomography of concrete using shear waves low-frequency phased antenna arrays. In Proceedings of the 17th world conference on nondestructive testing (Vol. 25, no. 28.10, p. 2008)

6. Büyüköztürk O (1998) Imaging of concrete structures. NDT \& E Int 31(4):233-243

7. Choi H, Bittner J, Popovics JS (2016) Comparison of ultrasonic imaging techniques for full-scale reinforced concrete. Transp Res Rec 2592(1):126-135

8. Edwards, L. and Mason, Q., 2011. Evaluation of nondestructive methods for determining pavement thickness. Engineer research and development center Vicksburg MS geotechnical and structures lab

9. FHWA (2014) Development of phased-array ultrasonic testing acceptability criteria (phase I). Publication no.: HRT-14-074

10. Flaherty, J.J., Erikson, K.R. and Van Lund, M., Magnaflux Corp, 1970. Synthetic aperture ultrasonic imaging systems. US Patent 3,548,642

11. Hoegh K, Khazanovich L (2015) Extended synthetic aperture focusing technique for ultrasonic imaging of concrete. NDT \& E Int 74:33-42

12. Hoegh K, Khazanovich L, Yu HT (2011) Ultrasonic tomography for evaluation of concrete pavements. Transp Res Rec 2232(1):85-94

13. Kaiser H, Karbhari VM, Sikorsky C (2004) Non-destructive testing techniques for FRP rehabilitated concrete. II: an assessment. Int J Mater Prod Technol 21(5):385-401

14. Kewalramani MA, Gupta R (2006) Concrete compressive strength prediction using ultrasonic pulse velocity through artificial neural networks. Autom Constr 15(3):374-379

15. Kohl C, Krause M, Maierhofer C, Wöstmann J (2005) 2D-and 3D-visualisation of NDT-data using data fusion technique. Mater Struct 38(9):817-826

16. Lin S, Shams S, Choi H, Azari H (2018) Ultrasonic imaging of multi-layer concrete structures. NDT \& E Int 98:101-109

17. Lovelace B, Wells J, Rand K, Nelson W, Stuber C (2017) Phased array ultrasonic steel corrosion mapping for bridges and ancillary structures (No. MN/RC 201733). Dept. of Transportation. Research Services \& Library, Minnesota

18. Mielentz F (2008) Phased arrays for ultrasonic investigations in concrete components. J Nondestruct Eval 27(1-3):23-33

19. MIRA 2018. A1040 - Ultrasonic low-frequency tomography. Operation manual, Acoustic Control Systems (ACS Group), Germany.

20. Perlin LP, and Pinto RCA (2013) Ultrasonic tomography in concrete. Rev Ibracon Estrut Mater 6(2):246-69

21. Poorarbabi A, Ghasemi M, Moghaddam MA (2020) Concrete compressive strength prediction using non-destructive tests through response surface methodology. Ain Shams Eng J https://doi.org/10.1016/j.asej.2020.02.009

22. Popovics JS, Roesler JR, Bittner J, Amirkhanian AN, Brand AS, Gupta P and Flowers K (2017) Ultrasonic imaging for concrete infrastructure condition assessment and quality assurance. Series No. 17-011, Research Report No. FHWA-ICT-17-007, Illinois Center for Transportation/llinois Department ofTransportation, Illinois

23. Salles L, Balbo JT, Khazanovich L (2017) Non-destructive ultrasonic tomography for concrete pavement evaluation: signal processing and image analysis of crucial parameters. Rev Ibracon Estrut Mater 10(6):1182-1191

24. Schickert M (2005) Progress in ultrasonic imaging of concrete. Mater Struct 38(9):807-815

25. Schickert M, Krause M, Müller W (2003) Ultrasonic imaging of concrete elements using reconstruction by synthetic aperture focusing technique. J Mater Civ Eng 15(3):235-246

26. Zatar W, Nguyen H (2017) Condition assessment of ground-mount cantilever weathering-steel overhead sign structures. J Infrastruct Syst 23(4):05017005

\section{Publisher's Note}

Springer Nature remains neutral with regard to jurisdictional claims in published maps and institutional affiliations. 